\title{
Non-thermal phenomena in galaxy clusters: seeing beyond the tip of the iceberg
}

\author{
Rossella Cassano* \\ INAF-Osservatorio di Radioastronomia, via P.Gobetti, 101, 40129, Bologna, Italy \\ E-mail: rcassanodira.inaf.it
}

Giant radio halos and relics are diffuse, Mpc-sized, synchrotron radio sources observed in a fraction of galaxy clusters. They probe the energy content and properties of relativistic particles and magnetic fields in galaxy clusters and their imprint on cluster formation and evolution. They are found in merging clusters, suggesting that they are generated as a result of the dissipation of gravitational energy during the hierarchical sequence of mergers that leads to the formation of clusters themselves. The properties of radio relics suggest a connection with large scale shocks that cross the intra-cluster medium (ICM) during mergers and that may accelerate locally injected electrons or reaccelerate pre-existing energetic electrons, while $\mathrm{RH}$ likely trace gigantic turbulent regions in the ICM, where relativistic electrons can be reaccelerated through scattering with magnetohydrodynamic turbulence. Nowadays statistical information about the presence (and absence) of diffuse Mpc-scale emission in galaxy clusters is available only for very massive clusters, those with $M_{500} \gtrsim 5.5 \times 10^{14} M_{\odot}$, which are however the rarest in the Universe. An interesting question is whether or not we are detecting just the tip of the iceberg of these phenomena. Future surveys, with LOFAR and SKA1, will explore low massive systems, $M_{500} \sim 10^{14} M_{\odot}$, that are one hundred time more numerous than those accessible with present facilities. How common is diffuse emission in clusters depends on theoretical models. In this contribution, we use Monte Carlo simulations, that combine turbulent-acceleration physics and the generation of secondaries in the ICM, to calculate the occurrence of radio halos in the Universe, their spectral properties and connection with properties of the hosting clusters at different cosmic epochs. Based on our study LOFAR and SKA1-LOW have the potential to increase the present number of cluster with radio halos by a factor of $\sim 20$ and $\sim 80$, respectively. Remarkably these surveys will be sensitive not only to radio halos in less massive clusters, but will have the potential to unveil the formation of radio halos in higher redshift systems (up to $z \sim 1$ ).

EXTRA-RADSUR2015 (*)

20-23 October 2015

Bologna, Italy

(*) This conference has been organized with the support of the Ministry of Foreign Affairs and International Cooperation, Directorate General for the Country Promotion (Bilateral Grant Agreement ZA14GR02 - Mapping the Universe on the Pathway to SKA)

\footnotetext{
* Speaker.
} 


\section{Introduction: non-thermal phenomena and cluster-cluster mergers}

Galaxy clusters are the largest gravitationally bound structures in the Universe, containing the equivalent mass of $\sim 10^{14}-10^{15} M_{\odot}$ in $2-3 \mathrm{Mpc}$ size. They are made especially by dark matter, that account for a $70-80 \%$ of the cluster mass, and whose presence can be investigated in the optical/near-IR bands through the study of the galaxy velocity distribution in the clusters and through weak-lensing analysis (e.g., Łokas \& Mamon 2003; Clowe et al. 2006). The largest baryonic component in galaxy clusters is in the form of the so-called Intra Cluster Medium (ICM), an hot and low density gas $\left(T \sim 10^{8}{ }^{\circ} \mathrm{K}, n_{e} \sim 10^{-3}-10^{-4} \mathrm{~cm}^{-3}\right)$ emitting in X-rays via thermal bremsstrahlung that account for $\sim 15-20 \%$ of the total cluster mass, while the remaining baryonic component is made by cluster galaxies, that made just few $\%$ of the total cluster mass. Observations of galaxy clusters in the radio band show the presence of diffuse synchrotron radiation from the ICM of merging galaxy clusters. These sources prove the existence of cosmic ray electrons and magnetic fields in the ICM and pose fundamental question about $i$ ) the origin of these components; ii) the impact of these components on the thermal ICM (microphysics); iii) the connection between these components and the cluster dynamics and evolution. This contribution will be focus mainly on iii).

Galaxy clusters form via a hierarchical sequence of mergers of smaller clusters and continuum accretion of unbound matter. Mergers between clusters are the most energetic phenomena since the big-bang, with a release of a gravitational potential energy of $\sim 10^{63}-10^{64}$ ergs during one cluster crossing time ( $\sim 1 \mathrm{Gyr})$. During such events shock waves and random vortical flows, if not turbulence, are produced in the ICM (e.g., Kulsrud et al. 1997; Norman \& Bryan 1999; Ricker \& Sarazin 2001). The bulk of the gravitational energy associated with the collision will be released as thermal energy in the final system (e.g., Kravtsov \& Borgani 2012), while another fraction can be channeled into non-thermal plasma components, i.e., relativistic particles and magnetic fields in the ICM (e.g., Brunetti \& Jones 2014).

Cluster-scale ( $\sim \mathrm{Mpc}$-scale) diffuse synchrotron emission is frequently found in merging galaxy clusters in the form of so-called giant radio halos (hereafter RH), apparently unpolarized synchrotron emission associated with the cluster X-ray emitting regions, and giant radio relics, elongated and often highly polarized synchrotron sources typically seen in the clusters outskirts (e.g., Feretti et al. 2012, for an observational review). The properties of radio relics suggest a connection with large scale shocks that cross the ICM during mergers and that may accelerate locally injected electrons or reaccelerate pre-existing energetic electrons, while RH likely trace gigantic turbulent regions in the ICM, where relativistic electrons can be reaccelerated through scattering with merger-induced MHD turbulence (e.g., Brunetti \& Lazarian 2007; Brüggen et al. 2012).

Radio and X-rays observations of galaxy clusters allowed to establish a clear connection between the cluster dynamics, constrained through the use of the X-ray data, and the presence of cluster-scale diffuse synchrotron radio emission (e.g., Buote 2001; Govoni et al. 2004; Cassano et al. 2010a). This contribution will be focused on giant RH. In the last decades, radio observations of statistical samples of galaxy clusters have shown that RHs are not ubiquitous, only $\sim 20-30 \%$ of the X-ray luminous $\left(L_{X}(0.1-2.4 \mathrm{keV}) \geq 5 \times 10^{44} \mathrm{erg} / \mathrm{s}\right)$ clusters host a RH (e.g., Venturi et al. 2008; Kale et al. 2015), while the fraction of clusters with RH becomes larger in SZ-selected clusters (e.g., Basu 2012; Cassano et al. 2013; Sommer \& Basu 2014; Cuciti et al. 2015). These 


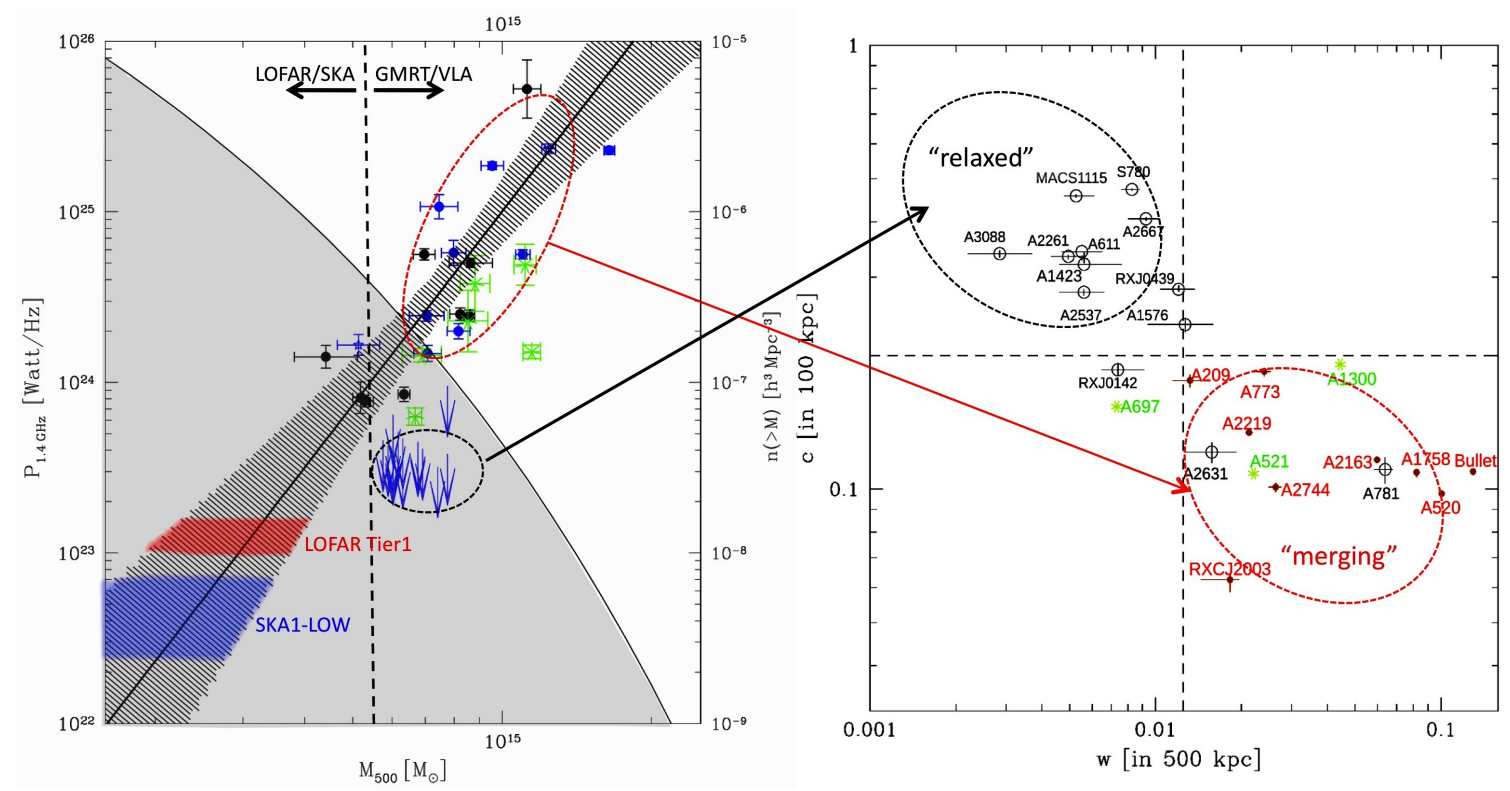

Figure 1: Left panel: RH power $P_{1.4}$ vs cluster mass $M_{500}$ (adapted from Cassano et al. 13). Symbols are, clusters belonging to the GMRT RH Survey (Venturi et al. 2008, and ref. therein): RH and upper limits (in blue), RH with ultra-steep spectra (USSRH, in green) and clusters with RH from the literature (in black). The best-fit relation to giant RH (black line) is reported together with its 95\% confidence region (shadowed regions). The $z=0.25$ galaxy cluster mass function is also reported (black curve/grey region; Jenkins et al. 2001). The red and blue region show the typical sensitivities of LOFAR and SKA1-LOW observations (assuming a spectral index $\alpha=1.3$ ). The dashed vertical line at $M_{500}=5.5 \times 10^{14} M_{\odot}$ divides the diagram into two regions: on the right side, is the place of the more massive and rarest objects in the Universe, those explored by present radio facilities (GMRT/VLA); on the left side, we find the less massive and more abundant objects, largely unexplored in radio and that will be the territory of LOFAR and SKA1. Right panel: distribution of clusters with $M_{500} \gtrsim 5.5 \times 10^{14} M_{\odot}$ in the merger diagnostic diagram: concentration parameter, $c$, versus the emission centroid shift, $w$ (Cassano et al. 2013 for details). Clusters with RH (red points and green asterisks) and clusters without RH (black points) clearly occupy different region, the upper left panel is the place of relaxed clusters, whereas the bottom right region is the place of merging clusters.

studies were possible thanks to the GMRT RH Survey (and its extension; e.g., Venturi et al. 2007, 2008; Kale et al. 2013, 2015) that is a deep radio survey dedicated to the search of RH in a X-ray selected sample of clusters in the redshift range $z \sim 0.2-0.35$. The most important outcome of this survey is illustrated in Fig.1: RH and non-RH clusters are clearly separated in the $P_{1.4}-M_{500}$ diagram $^{1}$ (left panel); this separation is related to their dynamical status, with $\mathrm{RH}$ associated to dynamically disturbed clusters and clusters without RHs statistically more "relaxed" (right panel) (Cassano et al. 2010a, 2013). This connection between RHs and merging clusters has been further supported by a number of independent studies (e.g., Rossetti et al. 2011; Wen \& Han 2015; Parekh et al. 2015; Mantz et al. 2015; Yuan et al. 2015). In Fig. 1 (left panel) we also show the cluster mass function at $z \sim 0.25$ (the average redshift of GMRT clusters), in the form of $n(>M)$ that is

\footnotetext{
${ }^{1}$ This radio bi-modality was first put forward using the same cluster sample in the $P_{1.4}-L_{X}$ diagram (e.g., Brunetti et al. 2007, 2009)
} 
the number density of clusters above a given mass (values of the mass function can be read on the right y-axis; Jenkins et al. 2001). It is thus clear, that nowadays statistical information (collected mainly with VLA and GMRT pointed observations of selected sample of galaxy clusters) about the presence (and absence) of diffuse Mpc-scale emission in galaxy clusters is available only for very massive clusters, those with $M_{500} \gtrsim 5.5 \times 10^{14} M_{\odot}$, which are the rarest in the Universe. An interesting open question that immediately arises is whether or not we are seeing the tip of the iceberg. How many RH await discovery? Future surveys, for instance with LOFAR and SKA1-LOW, will explore low massive systems, $M_{500} \sim 10^{14} M_{\odot}$, that are about one hundred time more numerous than those observable by present facility (see mass function in Fig. 1, left panel). However, going to lower masses does not necessary implies that much more RH will be discovered, this is something strictly dependent on the underlying theoretical model.

According to turbulence-acceleration models the formation history of RHs depends on the cluster merging rate throughout cosmic epochs and on the mass of the hosting clusters themselves, which ultimately sets the energy budget that is available for the acceleration of relativistic particles. A key expectation is that RHs should preferentially be found in massive objects undergoing energetic merging events, whereas they should be rarer in less massive merging-systems. The reason making RHs less common in smaller systems is that the acceleration mechanism in the case of less energetic mergers is expected to generate increasingly steep spectra which become under luminous at higher frequencies (e.g., Cassano et al. 2006, 2010b). This theoretical conjecture is consistent with the observed radio bimodality in galaxy clusters and its connection to cluster dynamics (Fig. 1), it is also supported by the discovery of RHs with very-steep spectrum (e.g., Brunetti et al. 2008; Dallacasa et al. 2009).

The generation of secondary particles due to inelastic collisions between relativistic protons and thermal protons in the ICM is another mechanism for the generation of cluster-scale diffuse emission (e.g., Dennison 1980; Blasi \& Colafrancesco 1999). Studies in the radio and gamma-rays suggest that the contribution to RHs due to this latter mechanism is sub-dominant (Ackermann et al. 2010; Brunetti et al. 2012), however in more relaxed clusters it is expected to produce diffuse radio sources, called "off state" halos, fainter than classical RHs (e.g., Brunetti \& Lazarian 2011; Brown et al. 2011; Donnert et al. 2013).

In this contribution, we will adopt the theoretical framework described above and use Monte Carlo simulations, that combine turbulent-acceleration physics and the generation of secondaries in the ICM, to calculate the occurrence of RHs in the Universe and their spectral properties. These simulations provide a physically motivated way to model the connection between RHs (and their characteristics) and the thermodynamical properties and mass of the hosting clusters at different cosmic epochs. Predictions for LOFAR and SKA1-LOW will be discussed.

\section{Modeling the cosmological evolution of giant radio halos in galaxy clusters}

In this Section we provide a summary of the theoretical framework (a more detailed description can be found in Cassano \& Brunetti 2005; Cassano et al. 2006, 2010b, 2012) and of the most important implications for RH statistical properties and connection with the host clusters. We model the formation and evolution of two populations of cluster RHs: (i) "turbulent" halos generated in merging clusters by turbulent re-acceleration of relativistic particles, and (ii) "off-state" 
halos generated by secondary electrons in more relaxed clusters. We follow a simplified approach based on two separate cluster radio-populations. Specifically, we assume that those clusters where turbulence is not sufficient to generate RHs emitting at the observing frequency, $v_{o}$, host "off-state" halos. We assume that presently observed giant RHs, those following the RH power - cluster mass correlation (see Fig.1, left panel), are mainly driven by turbulent re-acceleration in merging clusters. Whereas the radio power of clusters hosting "off-state" halos is constrained by using limits derived for "radio quiet" galaxy clusters (see upper limits in Fig.1, left panel; Brunetti et al. 2007; Brown et al. 2011).

We model the properties of the "turbulent" and "off-state" halos and their cosmic evolution by means of a Monte Carlo approach, which is based on the semi-analytic model of Lacey \& Cole (1993; i.e., extended Press \& Schechter 1974) to describe the hierarchical process of formation of galaxy cluster dark matter halos. The merger history of a synthetic population of galaxy clusters is followed back in time and the generation of the turbulence in the ICM is estimated for each merger identified in the merger trees. It is assumed that turbulence is generated in the volume swept by the subcluster infalling into the main cluster and that a fixed fraction $(\sim 0.1-0.3)$ of the PdV work done by this subcluster goes into MHD turbulence, which in turn becomes available for particle acceleration on Mpc-scale. We do not follow directly the process of magnetic field growth and amplification in the ICM, but this is anchored to the evolution of the host cluster, assuming a scaling between the mean rms magnetic field $\langle B\rangle$ and the virial cluster mass, $M_{v},\langle B\rangle \propto M_{v}^{b}$, with $b \gtrsim 0.5$ (e.g., Dolag et al. 2002).

The most important expectation of turbulent re-acceleration scenarios is that the synchrotron spectrum of RHs should become gradually steeper above a frequency, $v_{s}$, that is determined by the competition between acceleration and energy losses and which is connected to the energetics of the merger events that generate the halos (e.g., Fujita et al. 2003; Cassano \& Brunetti 2005). The frequency $v_{s}$ depends on the acceleration efficiency, $\chi$, and on $\langle B\rangle$, as $v_{s} \propto\langle B\rangle \chi^{2} /\left(\langle B\rangle^{2}+B_{c m b}^{2}\right)^{2}$ (e.g., Cassano et al. 2006, 2010b $)^{2}$. Monte Carlo simulations of cluster formation histories allow to estimate the rate of turbulence-generation during mergers and to evaluate $\chi$ and $v_{s}$ and their dependence on cluster mass, redshift, and merger parameters in a statistical sample of synthetic clusters. The ensuing RH population is characterized by a complex mixture of sources with different spectra, with massive (and hot) clusters that have a tendency to generate halos with spectra, measured between two frequencies, that are flatter than those in less massive systems. To estimate the occurrence of RHs in surveys at different observing radio frequencies we assume that only those halos with $v_{s} \geq v_{o}$ can be observable, $v_{o}$ being the observing frequency. On the base of simple energy arguments, giant RHs with $v_{s} \geq 1 \mathrm{GHz}$ are generated in connection with the most energetic merger-events in the Universe, since only these mergers can generate enough turbulence on Mpc-scales and potentially produce the acceleration rate that is necessary to maintain the relativistic electrons emitting at these frequencies (Cassano \& Brunetti 2005). As a consequence, it is expected that the fraction of clusters with RH increases with the cluster mass, since more massive clusters are more turbulent and thus are more likely to host a turbulent RH. A first observational evidence in this direction has recently been published and it is based on the analysis of radio observations of a mass (SZ)-selected sample of galaxy clusters (see Cuciti et al. 2015); the occurrence

\footnotetext{
${ }^{2} B_{c m b}=3.2(1+z)^{2} \mu \mathrm{G}$ is the equivalent magnetic field of the cosmic microwave background (CMB) radiation
} 


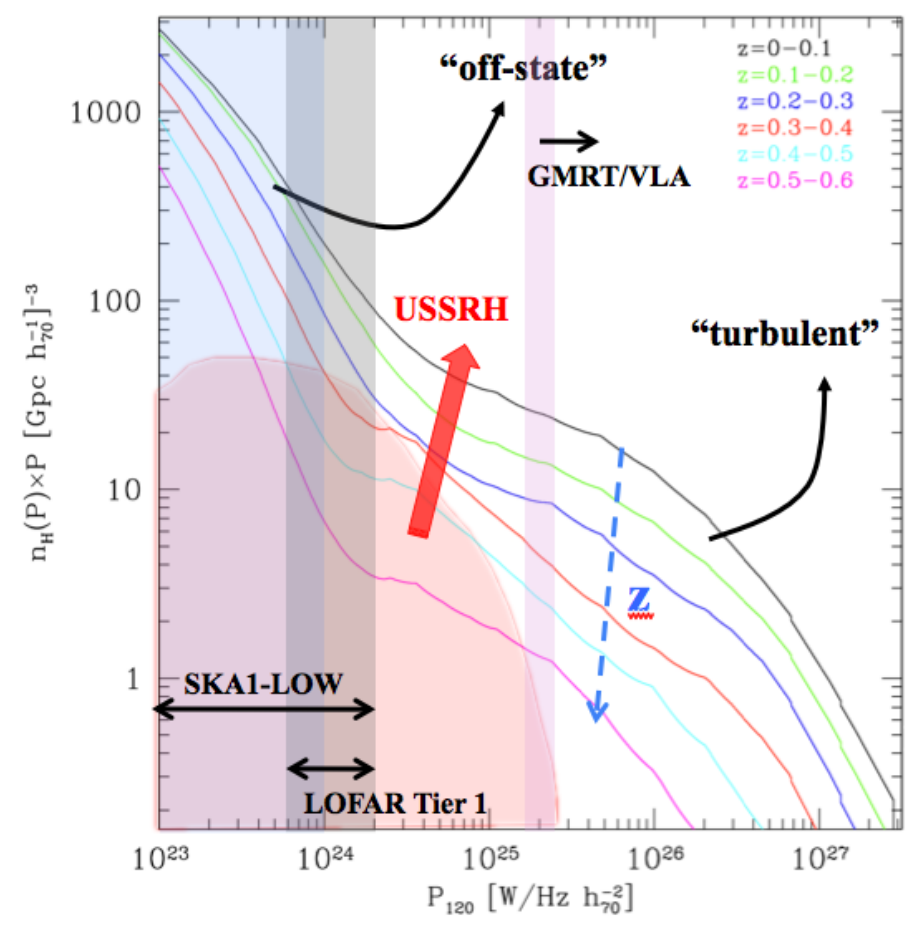

Figure 2: Total RHLFs at $120 \mathrm{MHz}$, obtained by combining the contributions from "turbulent" and from "off-state" (hadronic) halos, are reported at different redshifts (see figure legenda). The local $(z=0.05)$ RHLF of USSRH is also highlighted (shaded pink region). The expected sensitivities of SKA1-LOW and LOFAR are shown for RH at $z \sim 0.05$ (shaded vertical regions).

of RH was found to increase from $30 \%$ in clusters with mass in the range $M_{500} \sim 6-8 \times 10^{14} M_{\odot}$ up to $70-80 \%$ in clusters with $M_{500} \geq 8 \times 10^{14} M_{\odot}$.

For similar energy arguments RH with lower values of $v_{s}$, or with ultra-steep radio spectra $\left(\mathrm{USSRH}^{3}\right)$, must be more common, since they can be generated in connection with less energetic phenomena, e.g., major mergers between less massive systems or minor mergers in massive systems, that are more common in the Universe. The existence of USSRH implies that the occurrence of RH increases at low radio frequency (e.g., Cassano et al. 2006). A prototype example of USSRH was discovered in the galaxy cluster Abell 521 (Brunetti et al. 2008; Dallacasa et al. 2009) and some other examples exist in the literature (e.g., Cassano et al. 2013 and references therein).

Contrary to turbulent halos, "off-state" halos are expected with power-law spectra with fairly similar slopes, independently of the cluster mass. Consequently, surveying the sky at different radio frequencies and with appropriate sensitivities allows to disentangle these two populations. As an example, in Fig. 2 we show the total luminosity function of RH at $\sim 120 \mathrm{MHz}$ obtained by combining the contributions from "turbulent" RHs and from (purely hadronic) "off-state" halos and its redshift evolution (see figure legenda). Under our assumptions, "off-state" halos dominate the RHLF at lower radio luminosities where the RHLF due to turbulent RHs flattens. The contribution of USSRH to the local ( $z \sim 0.05$ ) RHLF at $120 \mathrm{MHz}$ is also highlighted (shaded red region) showing

\footnotetext{
${ }^{3}$ Operatively, in this paper we define USSRH as those halos with $\alpha>1.9$ between $250-600 \mathrm{MHz}$.
} 
that these systems contribute substantially to the RHLF at low radio powers, $P_{120} \sim 3-30 \times 10^{23}$ W/Hz. The expected sensitivity of SKA1-LOW and that of the LOFAR-Tier $1^{4}$ at $\sim 120 \mathrm{MHz}$ for clusters at $z \sim 0.05$ are also shown in comparison with that of typical GMRT/VLA observations (scaled here at $120 \mathrm{MHz}$, assuming a spectral index $\alpha \simeq 1.3$ ).

\section{Expectations for future surveys: from LOFAR to SKA1-LOW}

To derive the number of RH that can be detected in future surveys we estimated the minimum detectable flux of RH (integrated over a scale of $\sim 1 \mathrm{Mpc}$ ) as a function of the cluster redshift, $f_{\min }(z)$, considering two possible approaches, a brightness-based criterion and a flux-based criterion, which end up in two slightly different minimum flux thresholds (see Cassano et al. 2015 for more details). We consider both these estimates and derive the number of RHs with $f l u x \geq f_{\min }(z)$ in the redshift interval, $\Delta z=z_{2}-z_{1}$, as:
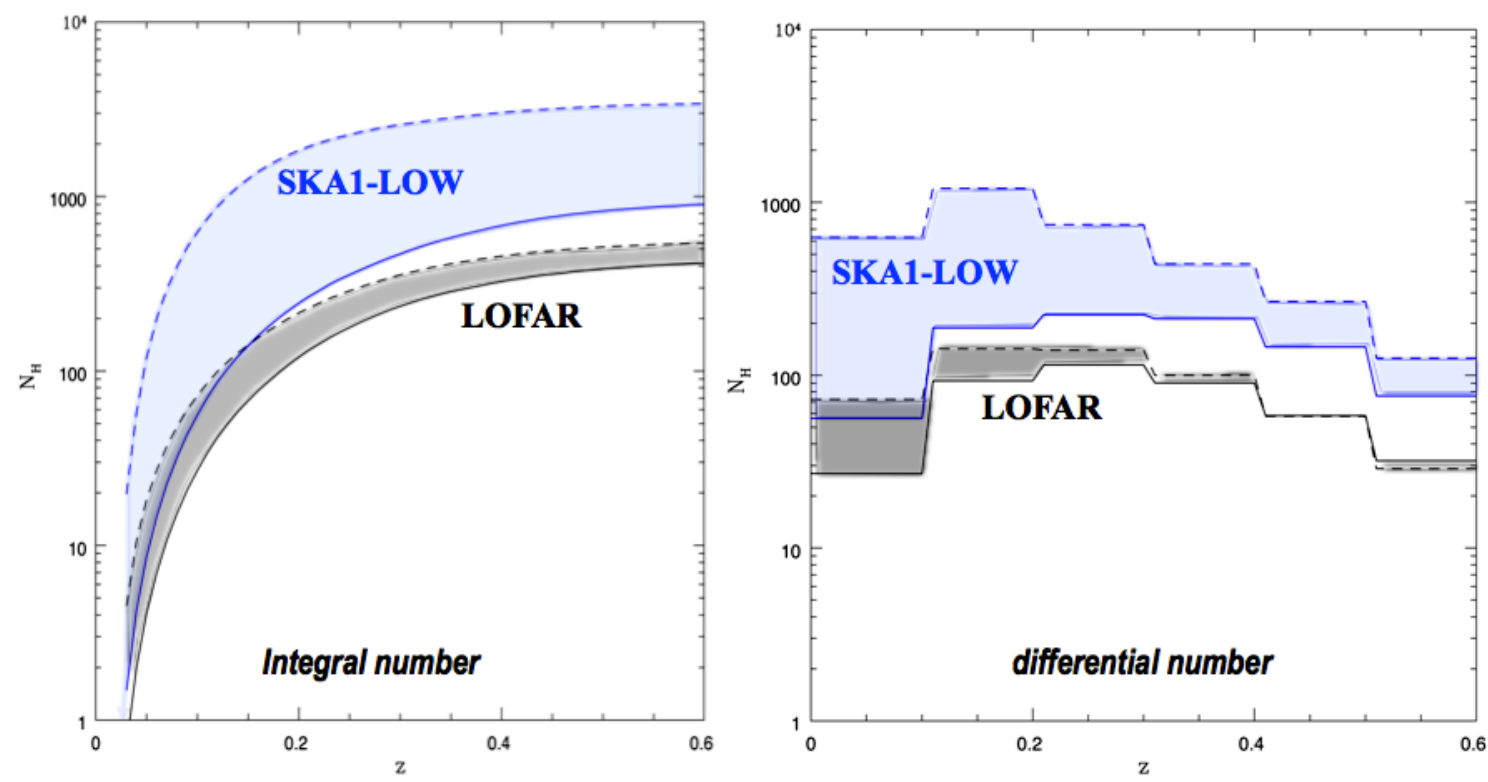

Figure 3: Left Panel: expected integral number (all-sky) of RHs as a function of redshift at $120 \mathrm{MHz}$. Right Panel: expected differential number of RH (all-sky) in redshift intervals at $120 \mathrm{MHz}$. In all plots, the shaded regions show the ranges obtained considering two possible methods to derive the RH detection limit (see text and Cassano et al. 2015) in the considered surveys: LOFAR ( $\mathrm{rms}=0.4 \mathrm{mJy} / \mathrm{beam}$ and $\theta_{b} \sim 25$ arcsec) and SKA1-LOW (rms $=20 \mu \mathrm{Jy} /$ beam, $\theta_{b}=10$ arcesc).

$$
N_{H}^{\Delta_{z}}=\int_{z=z_{1}}^{z=z_{2}} d z^{\prime}\left(\frac{d V}{d z^{\prime}}\right) \int_{P_{\min }\left(f_{\min }^{*}, z^{\prime}\right)} \frac{d N_{H}\left(P\left(v_{o}\right), z^{\prime}\right)}{d P\left(v_{o}\right) d V} d P\left(v_{o}\right)
$$

where $d N_{H}(z) / d P\left(v_{o}\right) d V$ is the RHLF and $d V / d z$ is the comoving volume element in the $\Lambda$ CDM cosmology (e.g., Carroll et al. 1992). In Fig.3 we show the number of RH expected in the LOFAR (black) and SKA1-LOW (blue) all-sky surveys (expectation for SKA1-MID and ASKAP can be

\footnotetext{
${ }^{4}$ Hewe we assume a LOFAR rms sensitivity $\simeq 200 \mu$ Jy/beam at 20 arcsec resolution (van Weeren et al. 2016a).
} 
find in Cassano et al. 2015), where we assume: rms $=0.4 \mathrm{mJy} / \mathrm{beam}$ and $\theta_{b} \sim 25 \operatorname{arcsec}$ for LOFAR 5 , and $\mathrm{rms}=20 \mu \mathrm{Jy} / \mathrm{beam}, \theta_{b}=10$ arcesc (confusion limited) for SKA1-LOW.

Given the typical RHLF (Fig.2) "off-state" halos are expected to contribute significantly to the total number of RHs at lower redshift $(z<0.3)$. LOFAR would mainly detect RH generated in turbulent merging clusters, while SKA1-LOW could start to test for the very first time the presence of hadronic halos in relaxed clusters ${ }^{6}$. In both cases, LOFAR and SKA1-LOW would be able to detect USSRH, with the number of these sources being larger in SKA1-LOW surveys thanks to the better sensitivity. The detection of a number of these USSRH is a powerful test for models (Brunetti et al. 2008).

SKA1-LOW and LOFAR could be able to detect up to $\sim 2600$ and $\sim 400$ respectively, on $3 \pi$ sr and out to $z \sim 0.6$, improving considerably the present number of know RH.

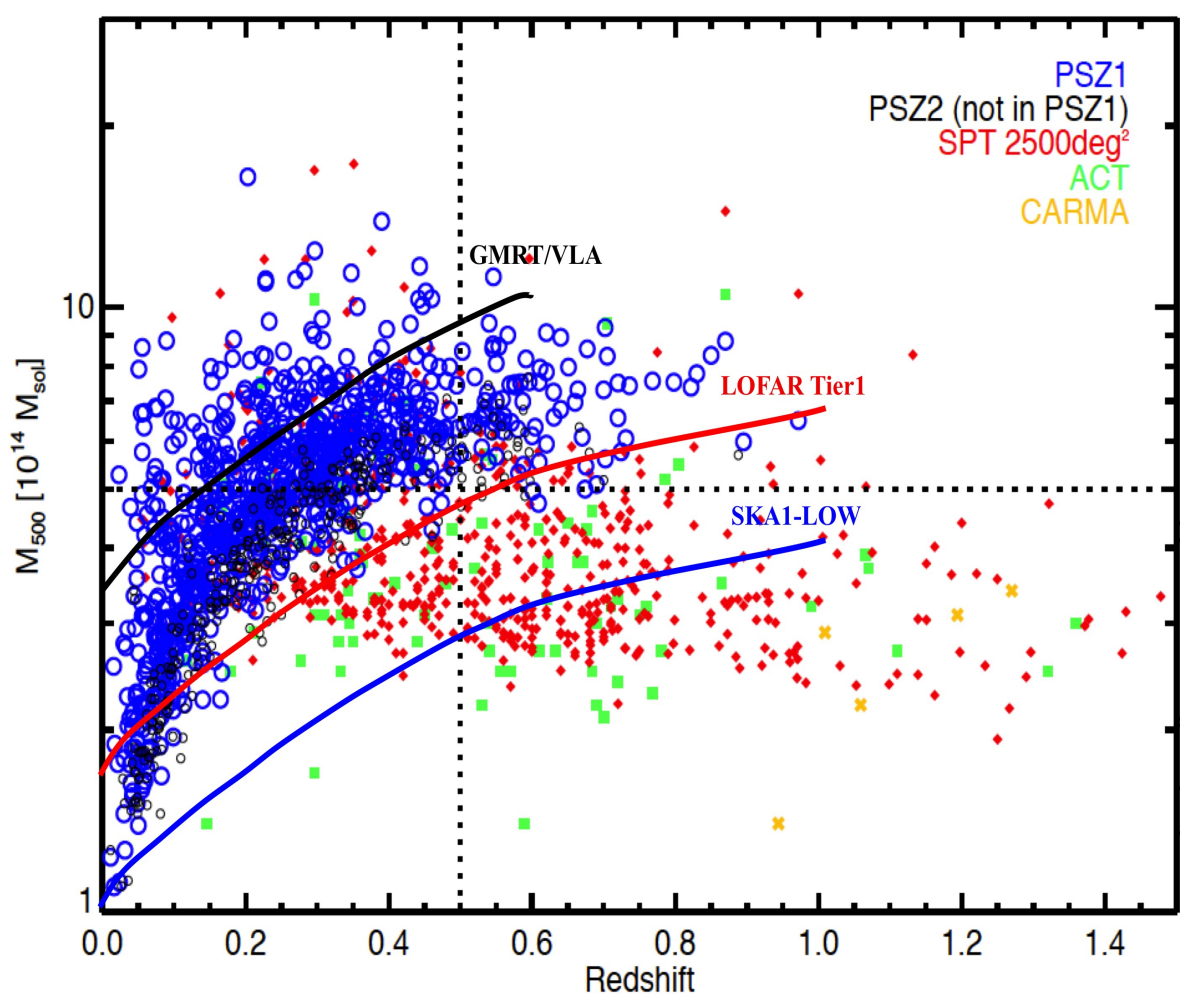

Figure 4: Distribution of SZ-detected clusters in the $M_{500}-z$ plane (taken from the SZ-cluster database, http://szcluster-db.ias.u-psud.fr). The sensitivity of present observations with VLA/GMRT and with LOFAR Tier1 and future observations with SKA1-LOW, are reported. They are derived by converting the radio power sensitivities into mass thresholds by assuming the radio power-cluster mass correlation (see Fig. 1, left panel).

\footnotetext{
$5_{\text {this }}$ is a conservative assumption, since pointed LOFAR observations obtained using configurations similar to the Tier 1 survey reached $\mathrm{rms}$ values $\sim 0.1 \mathrm{mJy} /$ beam at $\sim 5 \operatorname{arcsec}$ resolution and $\sim 0.2-0.3 \mathrm{mJy} / \mathrm{beam}$ at $\sim 20-30 \operatorname{arcsec}$ (van Weeren et al. 2016)

${ }^{6}$ Bonafede et al. (2015) recently claim the detection of a low-power RH in CIZA J1938.3+5409 (8 times below the radio-SZ correlation) that could represent the first detection of an "hadronic" halo.
} 


\section{Are there radio halos at high-z?}

In Fig.4 we report the distribution of SZ-detected clusters in the $M_{500}-z$ diagram (from the $S Z$ cluster database: http://szcluster-db.ias.u-psud.fr), together with the actual sensitivities to RH in these clusters by different radio telescopes (see figure legenda). It shows that while present observations with the VLA/GMRT are mostly sensitive to $\mathrm{RH}$ at lower and moderate redshifts ${ }^{7}$ (up to $z \sim 0.4$ ), future observations with LOFAR and SKA1-LOW have the potential to unveil RH in high redshift systems.

Theoretically, the generation of high-z RH is challenging due to the increase with $\mathrm{z}$ of the Inverse Compton losses of the radio emitting electrons $\left(d E / d t \propto(1+z)^{4}\right)$. Present models, based on semi-analytical calculations of cluster formation and turbulence generation, are only able to make trustworthy expectations for moderate-z $(z<0.5-0.6)$ clusters. This is because the PSbased Monte Carlo method starts to underproduce the number and merging rate of high-z clusters. This means that in the adopted formalism the formation of massive clusters is delayed (massive clusters start to form at lower $\mathrm{z}$ ) with respect to what is observed in cosmological simulations or predicted by more refined semi-analytic models (e.g., Giocoli 2012, 2013). This implies that the expectations presented for $\mathrm{RH}$ at $z \sim 0.5-0.6$ (Fig.3) should be taken as lower limits. More refined semi-analytical models to describe the formation and evolution of galaxy clusters (e.g., Giocoli 2012, 2013) combined with our formalism for non-thermal components will allow to obtain more reliable expectations at higher redshift (Cassano et al in prep).

\section{Acknowledgements}

It is a pleasure to thank my collaborators and colleagues (the list is too long), without their work and support this research would not have been possible.

\section{References}

[1] Ackermann, M., Ajello, M., Allafort, A., et al. 2010, ApJ, 717, L71

[2] Basu, K. 2012, MNRAS, 421, L112

[3] Blasi, P., \& Colafrancesco, S. 1999, Astroparticle Physics, 12, 169

[4] Bonafede, A., Feretti, L., Giovannini, G., et al. 2009, A\&A, 503, 707

[5] Bonafede, A., Brüggen, M., van Weeren, R., et al. 2012, MNRAS, 426, 40

[6] Bonafede, A., Intema, H., Brüggen, M., et al. 2015, MNRAS, 454, 3391

[7] Brown, S., Emerick, A., Rudnick, L., \& Brunetti, G. 2011, ApJ, 740, L28

[8] Brüggen, M., Bykov, A., Ryu, D., Röttgering, H. 2012, Space. Sci. Rev., 166, 187

[9] Brunetti, G., \& Jones, T. W. 2014, International Journal of Modern Physics D, 23, 30007

[10] Brunetti, G., \& Lazarian, A. 2007, MNRAS, 378, 245

\footnotetext{
${ }^{7}$ Only a few massive high-z $(z>0.5-0.6)$ clusters are known to host giant RH (e.g., Bonafede et al. 2009, 2012; van Weeren et al. 2009, 2014; Lindner et al. 2014).
} 
[11] Brunetti, G.; Venturi, T.; Dallacasa, D., 2007, et al. ApJ, 670, L5

[12] Brunetti G., Giacintucci S., Cassano R., et al., 2008, Nature 455, 944.

[13] Brunetti, G., Cassano, R., Dolag, K., \& Setti, G. 2009, A\&A, 507, 661

[14] Brunetti, G., \& Lazarian, A. 2011, MNRAS, 410, 127

[15] Brunetti, G., Blasi, P., Reimer, O., et al. 2012, MNRAS, 426, 956

[16] Buote, D. A. 2001, ApJL, 553, L15

[17] Carroll, S. M., Press, W. H., \& Turner, E. L. 1992, ARA\&A, 30, 499

[18] Cassano R. \& Brunetti G., 2005, MNRAS 357, 1313

[19] Cassano R., Brunetti G., Setti G., 2006, MNRAS 369,1577

[20] Cassano, R., Ettori, S., Giacintucci, S., et al. 2010a, ApJ, 721, L82

[21] Cassano, R., Brunetti, G., Röttgering, H. J. A., \& Brüggen, M. 2010b, A\&A, 509, A68

[22] Cassano, R., Brunetti, G., Norris, R. P., et al. 2012, A\&A, 548, A100

[23] Cassano, R., Ettori, S., Brunetti, G., et al. 2013, ApJ, 777, 141

[24] Cassano, R., Bernardi, G., Brunetti, G., et al. 2015, Advancing Astrophysics with the Square Kilometre Array (AASKA14), 73

[25] Clowe, D., Bradač, M., Gonzalez, A. H., et al. 2006, ApJL, 648, L109

[26] Cuciti, V., Cassano, R., Brunetti, G., et al. 2015, A\&A, 580, A97

[27] Dallacasa, D., Brunetti, G., Giacintucci, S., et al. 2009, ApJ, 699, 1288

[28] Dennison B., 1980, ApJ 239

[29] Dolag, K., Bartelmann, M., \& Lesch, H. 2002, A\&A, 387, 383

[30] Donnert, J., Dolag, K., Brunetti, G., \& Cassano, R. 2013, MNRAS, 429, 3564

[31] Feretti, L., Giovannini, G., Govoni, F., \& Murgia, M. 2012, A\&A Rev 20, 54

[32] Fujita Y., Takizawa M., Sarazin C.L., 2003, ApJ 584, 190

[33] Giocoli, C., Tormen, G., \& Sheth, R. K. 2012, MNRAS, 422, 185

[34] Giocoli, C., Marulli, F., Baldi, M., Moscardini, L., \& Metcalf, R. B. 2013, MNRAS, 434, 2982

[35] Govoni, F., Markevitch, M., Vikhlinin, A., et al. 2004, ApJ, 605, 695

[36] Jenkins, A., Frenk, C. S., White, S. D. M., et al. 2001, MNRAS, 321, 372

[37] Kale, R., Venturi, T., Giacintucci, S., et al. 2013, A\&A, 557, A99

[38] Kale, R., Venturi, T., Giacintucci, S., et al. 2015, A\&A, 579, A92

[39] Kravtsov, A. V., \& Borgani, S. 2012, ARA\&A, 50, 353

[40] Kulsrud, R. M., Cen, R., Ostriker, J. P., \& Ryu, D. 1997, ApJ, 480, 481

[41] Parekh, V., van der Heyden, K., Ferrari, C., Angus, G., \& Holwerda, B. 2015, A\&A, 575, A127

[42] Lacey, C., \& Cole, S. 1993, MNRAS, 262, 627

[43] Lindner, R. R., Baker, A. J., Hughes, J. P., et al. 2014, ApJ, 786, 49 
[44] Łokas, E. L., \& Mamon, G. A. 2003, MNRAS, 343, 401

[45] Mantz, A. B., Allen, S. W., Morris, R. G., et al. 2015, MNRAS, 449, 199

[46] Norman, M. L., \& Bryan, G. L. 1999, The Radio Galaxy Messier 87, 530, 106

[47] Press W.H., Schechter P., 1974, ApJ 187, 425

[48] Ricker, P. M., \& Sarazin, C. L. 2001, ApJ, 561, 621

[49] Rossetti, M., Eckert, D., Cavalleri, B. M., et al. 2011, A\&A, 532, A123

[50] Sommer, M. W., Basu, K. 2014, MNRAS, 437, 2163

[51] van Weeren, R. J., Röttgering, H. J. A., Brüggen, M., \& Cohen, A. 2009, A\&A, 505, 991

[52] van Weeren, R. J., Intema, H. T., Lal, D. V., et al. 2014, ApJ, 781, L32

[53] van Weeren, R. J., Brunetti, G., Brüggen, M., et al. 2016a, ApJ, 818, 204

[54] van Weeren, R. J., Williams, W. L., Hardcastle, M. J., et al. 2016b, ApJS 223, 2

[55] Venturi T., Giacintucci S., Brunetti G., et al. 2007, A\&A 463, 937

[56] Venturi, T., Giacintucci, S., Dallacasa, et al. 2008, A\&A, 484, 327

[57] Wen, Z. L., \& Han, J. L. 2015, MNRAS, 448, 2

[58] Yuan, Z. S., Han, J. L., \& Wen, Z. L. 2015, ApJ, 813, 77 